\title{
Rotationally Invariant Space-Time Trellis Codes with 4-D Rectangular Constellations for High Data Rate Wireless Communications
}

\author{
Corneliu Eugen D. Sterian, Cheng-Xiang Wang, Ragnar Johnsen, and Matthias Pätzold
}

\begin{abstract}
We demonstrate rotationally invariant space-time (ST) trellis codes with a 4-D rectangular signal constellation for data transmission over fading channels using two transmit antennas. The rotational invariance is a good property to have that may alleviate the task of the carrier phase tracking circuit in the receiver. The transmitted data stream is segmented into eight bit blocks and quadrature amplitude modulated using a 256 point 4-D signal constellation whose 2 -D constituent constellation is a 16 point square constellation doubly partitioned. The 4-D signal constellation is simply the Cartesian product of the 2-D signal constellation with itself and has 32 subsets. The partition is performed on one side into four subsets $A, B, C$, and $D$ with increased minimum-squared Euclidian distance, and on the other side into four rings, where each ring includes four points of equal energy. We propose both linear and nonlinear ST trellis codes and perform simulations using an appropriate multiple-input multiple-output (MIMO) channel model. The 4-D ST codes constructed here demonstrate about the same frame error rate (FER) performance as their 2-D counterparts, having however the added value of rotational invariance.
\end{abstract}

Index Terms: Antenna diversity, MIMO systems, multidimensional constellations, rotational invariance, space-time coded modulation, wireless communications.

\section{INTRODUCTION}

The term "space-time (ST) coding" has been coined by Tarokh et al. in their seminal paper [1] to mean a kind of radio channel coding that is performed across the spatial dimension as well as time to exploit the spatial diversity provided by using multiple transmit antennas. The basic idea, which is fully detailed also in a series of papers by the same research team [2]-[5], is to use more than a single transmit antenna so as to have at least two independently faded paths from the transmitter to the receiver. The receiver periodically measures the channel parameters, which are thus known to it, at least approximately, but not to the transmitter.

After Alamouti discovered a nice two transmit antenna combining scheme [6], the interest of researchers moved to the socalled "ST block codes". These block codes can provide full diversity, but no coding gain. Recently, in order to obtain also

Manuscript received December 3, 2003; approved for publication by Kyungwhoon Cheun, Division II Editor, May 10, 2004.

Corneliu Eugen D. Sterian is with the Polytechnic University of Bucharest, Romania, email: steriancorneliu@ hotmail.com.

Cheng-Xiang Wang, Ragnar Johnsen, and Matthias Pätzold are with the Department of Information and Communication Technology, Faculty of Engineering and Science, Agder University College, Grimstad, Norway, email: cheng.wang@hia.no; ragnar.johnsen@hia.no; matthias.paetzold@hia.no. a coding gain, Jafarkhani and Seshadri designed multidimensional ST codes with state transitions labeled with small block codes [7]. The schemes like those disclosed in [1] require that the radio channel be periodically measured in order to make the fading coefficients known to the receiver. To avoid this time consuming operation, Hochwald and Marzetta [8], Hochwald and Sweldens [9], and Hughes [10], [11] developed the theory of so-called "unitary ST modulation" that requires no coherent demodulation and therefore no knowledge of the channel parameters at the receiver. Nevertheless, recent papers [7], [12] show that the interest for schemes requiring the knowledge of the channel parameters by the receiver side has not diminished.

The theory of fading channels is largely available (see for instance [13], [14]). However, using these channels for mobile communication purposes is a permanent challenge to scientists. The search for new and better particular solutions for improving the performance of wireless communication systems over fading channels will certainly not slow down in the foreseeable future. In the last five years, a rich literature on channel modeling and coding systems flourished, including books [15]-[17]. There is already a large body of literature on ST codes. Some of the research papers attempt to generalize and subsume preceding work [18]. Nevertheless, it is not clear as yet which contributions have a bright future and which ones will be forgotten by lack of prospects. Therefore, we have not abandoned the line of investigation started by [1]. Actually, we also benefit in the present contribution from the big corpus of trellis coded modulation (TCM) literature, e.g., [19]-[21], and are heavily indebted to the work of Lee Fang Wei [22]. This work is also based on a particular method of 4-D TCM described in [23].

In order to obtain also rotational invariance, our approach was to develop the idea in [1] in the direction of a higher dimensionality of the signal space. To the best of our knowledge, no other research team has demonstrated rotationally invariant ST trellis codes as yet. Motivated by the known advantages of $2 \mathrm{~N}-\mathrm{D}$ over 2-D in the well studied case of TCM [22], [24], we considered a 4-D QAM signal set partitioned in such a way that the 4-D points in a 4-D subset are different in both the first and the second 2-D component points. As expected, the design of ST trellis codes with 4-D QAM is rather elaborated. Therefore, to facilitate the understanding, we describe it in a detailed manner.

The paper is organized as follows: In Section II, we consider the 2-D 16-QAM ST code with 16 states disclosed in [1] and design also other 16-, 32- and 64-state 2-D ST trellis codes that transmit $b=4$ bits per signaling interval. These ST codes will be used later as reference for our new 4-D rotationally invariant ST trellis codes. Section III describes the 4-D signal constella- 
tion and its partition. Section IV presents the general structure of an encoder that generates 32,64 , and generally $2^{\nu}$ states for $\nu \geq 5$, rotationally invariant 4-D ST trellis codes to transmit $b=4$ bits per signaling interval. Then, Section $\mathrm{V}$ demonstrates several examples of ST codes, both linear and nonlinear. Section VI gives the error performance simulation results compared to the 2-D 16-QAM ST codes considered in Section II as reference system. The MIMO channel simulation model for wireless communications that we used for all ST codes was developed in [25]. Using it, we recovered the results published in [1] for the 2-D 16-QAM ST code and this is, we believe, indicative of the fact that the two simulation models, Tarokh's and ours, while different, are nevertheless equivalent, at least for the purpose at hand. Finally, Section VII presents some conclusions.

\section{2-D SPACE-TIME 16-QAM TRELLIS CODES}

\section{A. 16-State 2-D Trellis Codes}

In [1], many ST trellis codes for 4-PSK and 8-PSK are demonstrated, and a single 16-QAM code with 16 states is given. The 16-QAM constellation (Fig. 18 in [1]) is reproduced here in Fig. 1 for convenience.

The input bit stream is segmented into blocks of $b=4$ bits denoted as $I 1_{n}, I 2_{n}, I 3_{n}$, and $I 4_{n}$. Such a block called quadribit is applied as input to a convolutional encoder with 16 states at discrete time $n$. Let us denote the current state of the convolutional encoder as $W 4_{n} W 3_{n} W 2_{n} W 1_{n}$, where $W 1_{n}, W 2_{n}, W 3_{n}$ and $W 4_{n}$ are binary variables, then, the states can be numbered from 0 to 15 using the relation

$$
W_{n}=8 W 4_{n}+4 W 3_{n}+2 W 2_{n}+W 1_{n} .
$$

Every transition from the current state $W_{n}$ to the next state $W_{n+1}$ is labeled with $Y_{n}^{(1)} Y_{n}^{(2)}$, where $Y_{n}^{(1)}$ and $Y_{n}^{(2)}$ are the 2D points from the 16-QAM constellation simultaneously transmitted by Antenna 1 and by Antenna 2, respectively, at discrete time $n$. We write the point labels using four binary variables $Y 1_{n}^{(i)}, Y 2_{n}^{(i)}, Y 3_{n}^{(i)}$ and $Y 4_{n}^{(i)}$ as follows:

$$
Y_{n}^{(i)}=8 Y 4_{n}^{(i)}+4 Y 3_{n}^{(i)}+2 Y 2_{n}^{(i)}+Y 1_{n}^{(i)} \quad i=1,2 .
$$

Tarokh et al. have designed their ST trellis codes in [1] using the following rules:

Rule 1: Transitions departing from the same state differ in the second symbol.

Rule 2: Transitions arriving at the same state differ at least in one symbol and, if possible, in both symbols.

The 16-state trellis code for the 16-QAM constellation given in Fig. 1 was obtained by Tarokh et al. from the block code $(00$, $111,22,39,4$ 4, 5 15, 6 6, 7 13, 8 8, 9 3, $1010,111,1212,13$ $7,1414,155$ ) of length 2 defined over the alphabet 16-QAM. This block code is optimal in the sense of product distance [1].

The 16-QAM trellis code with 16 states whose trellis diagram is given in [1, Fig. 19] has the block diagram shown in Fig. 2. As may be seen, this is just a modified form of a delay diversity scheme. For this code, transitions reaching the same state are

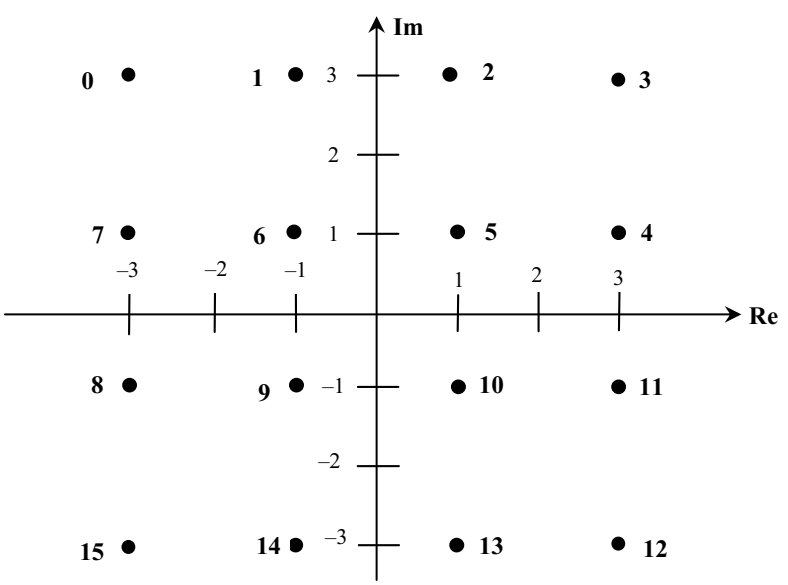

Fig. 1. The 16-QAM signal constellation with points labeled as in [1].

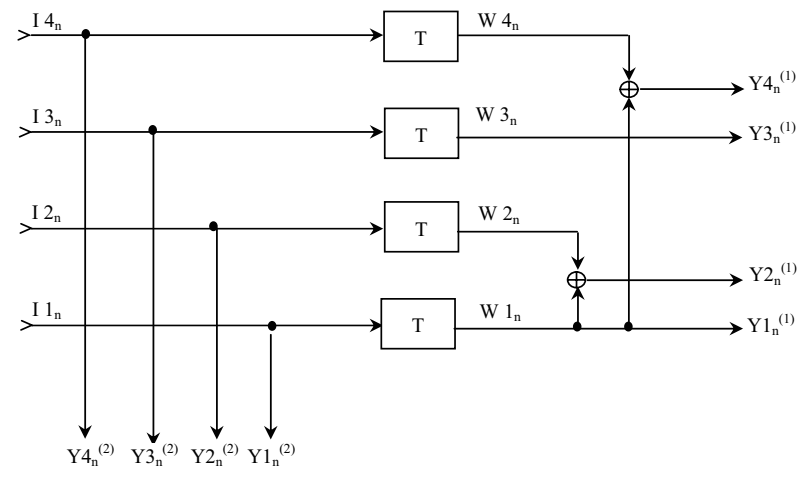

Fig. 2. 16-state, rate 4/8, ST convolutional encoder corresponding to the trellis diagram of Fig. 19 in [1].

assigned two symbols from which the first one is the same and the second one is different.

Let us now consider another labeling of the state transitions of a 16-state convolutional encoder such that the rule 2 sounds: Transitions arriving at the same state differ in both the first and the second symbol. The trellis diagram is the same as that given in [1, Fig. 19], but the labeling is different. The first part of the labels of all 16 transitions originating from the same state $W_{n}$ [see (1)] is as follows:

$$
\begin{aligned}
Y 1_{n}^{(1)} & =W 1_{n} \\
Y 2_{n}^{(1)} & =W 1_{n} \oplus W 2_{n} \\
Y 3_{n}^{(1)} & =W 3_{n} \\
Y 4_{n}^{(1)} & =W 1_{n} \oplus W 4_{n} .
\end{aligned}
$$

Now, with the binary variables $I 1_{n}, I 2_{n}, I 3_{n}$, and $I 4_{n}$ form the decimal number $I_{n}$ :

$$
I_{n}=8 I 4_{n}+4 I 3_{n}+2 I 2_{n}+I 1_{n} .
$$

The second part of the label of the transition from the current state $W_{n}$ to the next state $W_{n+1}$ produced by the current quadribit applied at the input is as written below:

$$
Y_{n}^{(2)}=W_{n}+I_{n} \quad \text { (modulo 16). }
$$




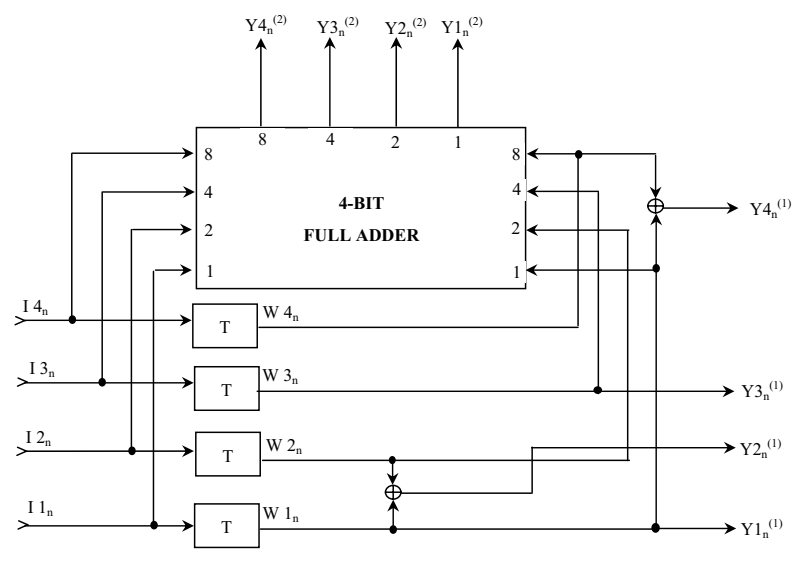

Fig. 3. New 16-state, rate 4/8, 2-D ST nonlinear convolutional encoder.

The block diagram of the corresponding (nonlinear) trellis encoder is given in Fig. 3.

Using the same design rules, we have also designed 32- and 64- state 2-D trellis codes.

\section{B. 32-State 2-D Nonlinear Trellis Code}

Denote the current state of the convolutional encoder as $W 5_{n} W 4_{n} W 3_{n} W 2_{n} W 1_{n}$, where $W 1_{n}, W 2_{n}, W 3_{n}, W 4_{n}$ and $W 5_{n}$ are binary variables. Let us number in decimal notation the states from 0 to 31 using the relation

$$
W_{n}=16 W 5_{n}+8 W 4_{n}+4 W 3_{n}+2 W 2_{n}+W 1_{n} .
$$

The first part of the label of all 16 transitions originating from the same state $W_{n}$ [see (6)] is as given below:

$$
\begin{aligned}
Y 1_{n}^{(1)} & =W 1_{n} \oplus W 5_{n} \\
Y 2_{n}^{(1)} & =W 1_{n} \oplus W 2_{n} \oplus W 5_{n} \\
Y 3_{n}^{(1)} & =W 3_{n} \\
Y 4_{n}^{(1)} & =W 1_{n} \oplus W 4_{n} \oplus W 5_{n} .
\end{aligned}
$$

As about the second part of the label of the state transitions, first form the following binary variables:

$$
\begin{aligned}
V 1_{n} & =W 1_{n} \oplus W 5_{n} \\
V 2_{n} & =W 2_{n} \\
V 3_{n} & =W 3_{n} \\
V 4_{n} & =W 4_{n} .
\end{aligned}
$$

Now, with the binary variables $V 1_{n}, V 2_{n}, V 3_{n}$, and $V 4_{n}$, form the decimal number $V_{n}$ according to

$$
V_{n}=8 V 4_{n}+4 V 3_{n}+2 V 2_{n}+V 1_{n}
$$

The second part of the label of the transition from the current state $W_{n}$ to the next state $W_{n+1}$ produced by the current quadribit applied at the input is

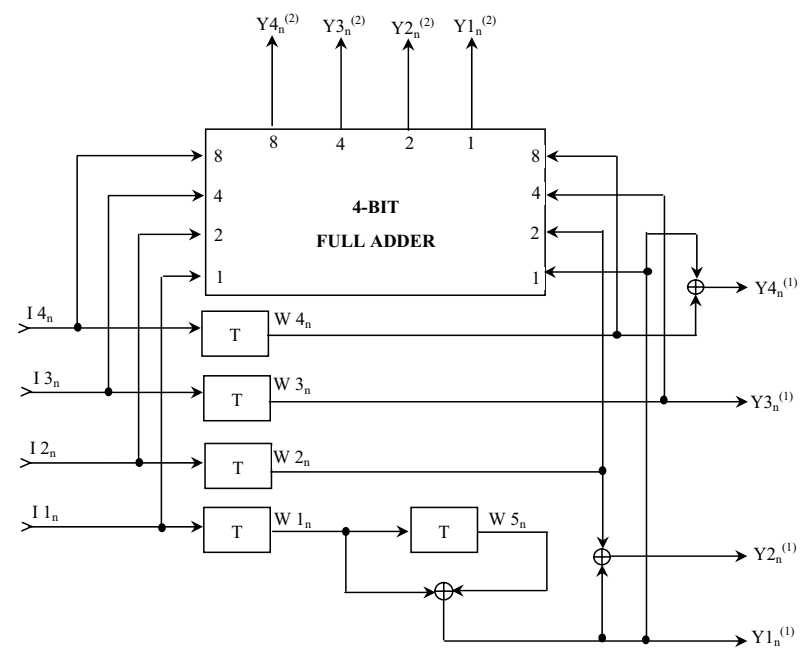

Fig. 4. 32-state, rate 4/8, 2-D ST nonlinear convolutional encoder.

$$
Y_{n}^{(2)}=V_{n}+I_{n} \quad(\text { modulo } 16) .
$$

The block diagram of the corresponding (nonlinear) trellis encoder is given in Fig. 4.

\section{64-State 2-D Nonlinear Trellis Code}

Denote the current state of the convolutional encoder as $W 6_{n} W 5_{n} W 4_{n} W 3_{n} W 2_{n} W 1_{n}$, where $W 1_{n}, W 2_{n}, W 3_{n}$, $W 4_{n}, W 5_{n}$, and $W 6_{n}$ are binary variables. Let us number in decimal notation the states from 0 to 63 using the relation

$$
\begin{aligned}
W_{n}= & 32 W 6_{n}+16 W 5_{n}+8 W 4_{n}+4 W 3_{n} \\
& +2 W 2_{n}+W 1_{n} .
\end{aligned}
$$

The first part of the label of all 16 transitions originating from the same state $W_{n}$ [see (11)] is as follows:

$$
\begin{aligned}
Y 1_{n}^{(1)} & =W 1_{n} \oplus W 5_{n} \\
Y 2_{n}^{(1)} & =W 1_{n} \oplus W 2_{n} \oplus W 5_{n} \oplus W 6_{n} \\
Y 3_{n}^{(1)} & =W 3_{n} \\
Y 4_{n}^{(1)} & =W 1_{n} \oplus W 4_{n} \oplus W 5_{n} .
\end{aligned}
$$

To describe the second part of the label of a state transition, form first the following binary variables:

$$
\begin{aligned}
V 1_{n} & =W 1_{n} \oplus W 5_{n} \\
V 2_{n} & =W 2_{n} \oplus W 6_{n} \\
V 3_{n} & =W 3_{n} \\
V 4_{n} & =W 4_{n} .
\end{aligned}
$$

Then, the second part of the label of the transition from the current state $W_{n}$ to the next state $W_{n+1}$ produced by the current quadribit applied at the input is given by (10). The block diagram of the corresponding (nonlinear) trellis encoder is given in Fig. 5. 


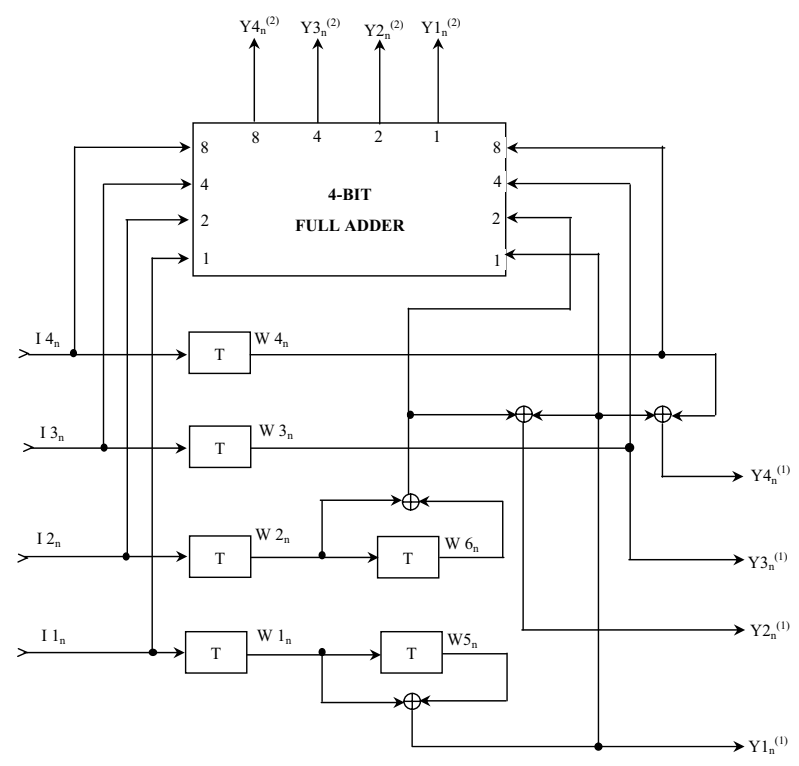

Fig. 5. 64-state, rate 4/8, 2-D ST nonlinear convolutional encoder.

\section{4-D SIGNAL CONSTELLATION AND ITS PARTITION}

We will consider in this paper the sixteen-point 2-D constellation shown in Fig. 6. It allows a data rate of four bits per channel use. The points of the 2-D signal constellation belong to a rectangular grid and have odd integer coordinates. In other words, if $Z$ is the set of integers, then the coordinates of the 2-D points belong to the set $\{2 Z+1\}^{2}$. We partition this infinite set into four 2-D subsets $A, B, C$, and $D$ according to

$$
\begin{aligned}
& A=\{4 Z+1\}^{2} \\
& B=\{4 Z+3\}^{2} \\
& C=\{4 Z+1\}\{4 Z+3\} \\
& D=\{4 Z+3\}\{4 Z+1\} .
\end{aligned}
$$

If we denote the minimum-squared Euclidian distance (MSED) of the set $\{2 Z+1\}^{2}$ as $\delta_{0}^{2}$, then the MSED of every subset $A, B, C$, and $D$ is $4 \delta_{0}^{2}$. A 4-D point is simply a concatenation of two 2-D points that will be successively transmitted by the same antenna.

Sixteen 4-D types may then be defined, each corresponding to a concatenation of two 2-D subsets, and denoted as $(A, A),(A, B), \ldots$, and $(D, D)$. The 164 -D types are grouped into four subsets such that the types within a subset are different of each other in both the first and the second 2-D component:

$$
\begin{aligned}
& S S_{0}=(A, A) \cup(B, B) \cup(C, C) \cup(D, D) \\
& S S_{1}=(A, C) \cup(B, D) \cup(C, B) \cup(D, A) \\
& S S_{2}=(A, B) \cup(B, A) \cup(C, D) \cup(D, C) \\
& S S_{3}=(A, D) \cup(B, C) \cup(C, A) \cup(D, B) .
\end{aligned}
$$

Note with reference to Fig. 6 that these four subsets are invariant under $90^{\circ}, 180^{\circ}$, and $270^{\circ}$ rotation. This property will greatly simplify the building of rotational invariance into the codes.

The sixteen-point 2-D constellation in Fig. 6 is also partitioned into four subsets called rings. Each ring includes four

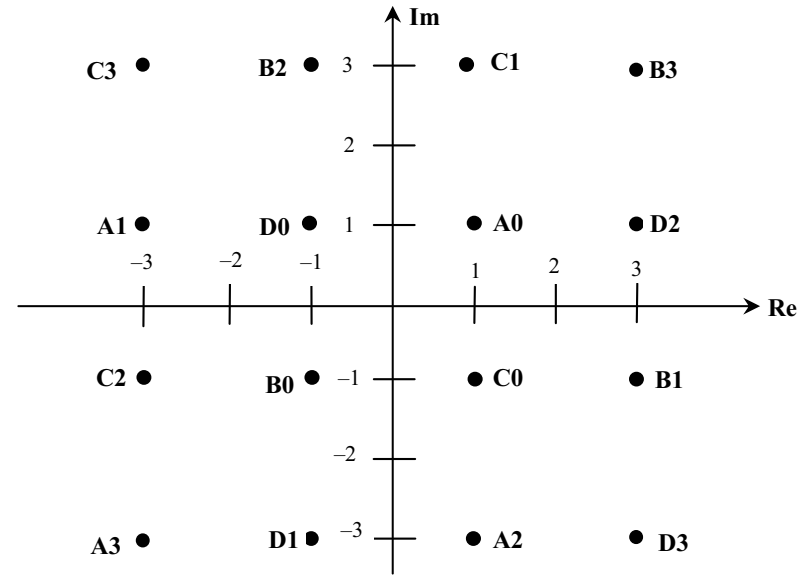

Fig. 6. Sixteen-point 2-D constellation partitioned into four subsets $(A, B, C, D)$ and into four rings $\left(R_{0}, R_{1}, R_{2}, R 3\right)$.

points of equal norm or energy such that a rotation of any point by $90^{\circ}, 180^{\circ}$, and $270^{\circ}$ produces the other three points of the same ring. Here, the norm of a point is defined as the squared distance to the origin. Therefore, the ring $R_{0}$ contains the points $A 0, B 0, C 0$, and $D 0$ of norm 2 , the ring $R_{1}$ contains the points $A 1, B 1, C 1$, and $D 1$ of norm 10 , the ring $R_{2}$ has points $A 2, B 2, C 2$, and $D 2$ of norm 10 , and the ring $R_{3}$ has points $A 3, B 3, C 3$, and $D 3$ of norm 18 . Using these rings, we form next a 256-point 4-D constellation partitioned into eight subsets called shells as follows:

$$
\begin{aligned}
& S H_{0}=\left(R_{0}, R_{0}\right) \cup\left(R_{1}, R_{1}\right) \\
& S H_{1}=\left(R_{0}, R_{1}\right) \cup\left(R_{1}, R_{0}\right) \\
& S H_{2}=\left(R_{0}, R_{2}\right) \cup\left(R_{2}, R_{0}\right) \\
& S H_{3}=\left(R_{0}, R_{3}\right) \cup\left(R_{3}, R_{0}\right) \\
& S H_{4}=\left(R_{1}, R_{2}\right) \cup\left(R_{2}, R_{1}\right) \\
& S H_{5}=\left(R_{1}, R_{3}\right) \cup\left(R_{3}, R_{1}\right) \\
& S H_{6}=\left(R_{2}, R_{3}\right) \cup\left(R_{3}, R_{2}\right) \\
& S H_{7}=\left(R_{2}, R_{2}\right) \cup\left(R_{3}, R_{3}\right) .
\end{aligned}
$$

The partition has been done in such a way that the 4-D points inside a given shell are different bf each other in both the first and the second 2-D constituent po $\equiv$

Combining the four subsets $S S D=0,1,2,3)$, defined by (15a)-(15d), with the eight shells $S H_{j}(j=0,1, \ldots, 7)$, we obtain 324 -D subsets $S_{m}(m=0,1, \ldots, 31)$, where the index $m$ is given by the relation

$$
m=4 j+k .
$$

Every one of the 32 subsets contains eight 4-D points that are different from any other one in both the first and the second 2-D component of it. We will use this signal constellation to transmit blocks of eight bits.

The average energy $E_{s}$ of a 2-D square signal constellation that allows transmitting $b$ bits per signal point is given by the formula [26]

$$
E_{s}=\left(\frac{2}{3}\right)\left(2^{b}-1\right)
$$


Hence, for $b=4$, it follows $E_{s}=10$. We will assume from now on that each coordinate of the signal constellation shown in Fig. 6 is contracted by the scale factor $\sqrt{E_{s}}=\sqrt{10}$ chosen so that the average energy of the constellation points is 1 . For instance, the point $A 0$ has coordinates $\left(\frac{\sqrt{10}}{10}, \frac{\sqrt{10}}{10}\right)$ instead of $(1,1)$.

\section{GENERAL STRUCTURE OF A 4-D SPACE-TIME ENCODER FOR SENDING FOUR BITS PER SIGNALING INTERVAL}

In [1], only the 2-D case is considered. However, the system model developed there and the performance criteria derived therefrom apply to the 4-D case as well. Assume that a frame of $N$ 4-D symbols is transmitted by a communication system having two transmit antennas and denote the corresponding point as $\mathbf{s}=P_{1}^{(1)} P_{1}^{(2)} P_{2}^{(1)} P_{2}^{(2)} \ldots P_{N}^{(1)} P_{N}^{(2)}$. Because of the channel noise, a maximum-likelihood receiver may decide erroneously in favor of another signal point, say $\mathbf{e}=$ $R_{1}^{(1)} R_{1}^{(2)} R_{2}^{(1)} R_{2}^{(2)} \ldots R_{N}^{(1)} R_{N}^{(2)}$, where, at discrete time $n$, the 4-D symbols $P_{n}^{(1)}$ and $P_{n}^{(2)}$ are simultaneously transmitted by Antenna 1 and by Antenna 2, respectively.

The receiver may be equipped with any number of antennas, but, for simplicity reasons, let us consider the case of only two antennas. For every frame of $N$ 4-D symbols, it is \$upposed that the receiver measures perfectly the path gains $\alpha_{i} \equiv \mathrm{m}$ transmit antenna $i(i=1,2)$ to receive antenna $j(j=\square$, . Thus, the receiver has ideal channel state information (CSI). Form now the matrix:

$\mathbf{B}(\mathbf{s}, \mathbf{e})=\left(\begin{array}{ccc}R_{1}^{(1)}-P_{1}^{(1)} & R_{2}^{(1)}-P_{2}^{(1)} \ldots R_{N}^{(1)}-P_{N}^{(1)} \\ R_{1}^{(2)}-P_{1}^{(2)} & R_{2}^{(2)}-P_{2}^{(2)} \ldots R_{N}^{(2)}-P_{N}^{(2)}\end{array}\right)$.

According to the rank criterion derived in [1], the matrix $\mathbf{B}(\mathbf{s}, \mathbf{e})$ has to be full rank for any code words $\mathbf{s}$ and $\mathbf{e}$. Clearly, in case of two transmit antennas, the full rank is 2 . The determinant criterion, which gives the coding advantage, puts as a design target to make the sum of all $2 \times 2$ principal cofactors of $\mathbf{A}(\mathbf{s}, \mathbf{e})=\mathbf{B}(\mathbf{s}, \mathbf{e}) \mathbf{B}^{*}(\mathbf{s}, \mathbf{e})$ as large as possible for any code words $\mathbf{s}$ and $\mathbf{e}$, where $\mathbf{B}^{*}(\mathbf{s}, \mathbf{e})$ is the Hermitian (transpose conjugate) of $\mathbf{B}(\mathbf{s}, \mathbf{e})$.

Now, in our construction, as we will see shortly, three out of eight input bits are uncoded. Therefore, in the trellis diagram, there are $2^{3}=8$ parallel transitions between every originating state $W_{n}$ and any corresponding next state $W_{n+1}$. It follows that the shortest uncommon portion of the two strings $\mathbf{s}$ and $\mathbf{e}$ has length one. It may be seen that our way to partition the 4-D constellation into subsets of eight 4-D points that are different in both the first and the second 2-D component of them guarantees that the rank criterion is satisfied. As about uncommon paths of lengths larger than one, the two design rules 1 and 2 of [1], given here in Section II for convenience, guarantee that the rank criterion is always satisfied.

In order to satisfy the determinant criterion as well, we have developed the following block code:

$(0$ 0, $129,22,331,44,525,66,727,88,921,1010,1123$, $1212,1317,1414,1519,1616,1713,1818,1915,2020$, $219,2222,2311,2424,255,2626,277,2828,291,3030$,
Table 1. Partition of the 256-point 4-D signal set into 32 subsets.

\begin{tabular}{|c|c|c|c|c|c|c|c|}
\hline \begin{tabular}{|c|c|}
$4-D$ \\
Subset
\end{tabular} & $I 6_{n}$ & $Y 5^{(i)}$ & $Y 4^{(i)}$ & $Y 3^{(i)}$ & $Y 2^{(i)}$ & $Y 1(i)$ & 4-D Type \\
\hline$S_{0}$ & $\begin{array}{l}0 \\
1\end{array}$ & 0 & 0 & 0 & 0 & 0 & \begin{tabular}{|l}
$(A 0, A 0),(B 0, B 0),(C 0, C 0),(D 0, D 0)$ \\
$(A 1, A 1),(B 1, B 1),(C 1, C 1),(D 1, D 1)$
\end{tabular} \\
\hline$S_{1}$ & $\begin{array}{l}0 \\
1 \\
1\end{array}$ & 0 & 0 & 0 & 0 & 1 & $\begin{array}{l}(A 0, C 0),(B 0, D 0),(C 0, B 0),(D 0, A 0) \\
(A 1, C 1),(B 1, D 1),(C 1, B 1),(D 1, A 1) \\
\end{array}$ \\
\hline$S_{2}$ & $\begin{array}{l}0 \\
1\end{array}$ & 0 & 0 & 0 & 1 & 0 & $\begin{array}{l}(A 0, B 0),(B 0, A 0),(C 0, D 0),(D 0, C 0) \\
(A 1, B 1),(B 1, A 1),(C 1, D 1),(D 1, C 1)\end{array}$ \\
\hline$S_{3}$ & $\begin{array}{l}0 \\
1\end{array}$ & 0 & 0 & 0 & 1 & 1 & $\begin{array}{l}(A 0, D 0),(B 0, C 0),(C 0, A 0),(D 0, B 0) \\
(A 1, D 1),(B 1, C 1),(C 1, A 1),(D 1, B 1)\end{array}$ \\
\hline$S_{4}$ & $\begin{array}{l}0 \\
1\end{array}$ & 0 & 0 & 1 & 0 & 0 & $\begin{array}{l}(A 0, A 1),(B 0, B 1),(C 0, C 1),(D 0, D 1) \\
(A 1, A 0),(B 1, B 0),(C 1, C 0),(D 1, D 0)\end{array}$ \\
\hline$S_{5}$ & $\begin{array}{l}0 \\
1\end{array}$ & 0 & 0 & 1 & 0 & 1 & $\begin{array}{l}(A 0, C 1),(B 0, D 1),(C 0, B 1),(D 0, A 1) \\
(A 1, C 0),(B 1, D 0),(C 1, B 0),(D 1, A 0) \\
\end{array}$ \\
\hline$S_{6}$ & $\begin{array}{l}0 \\
1\end{array}$ & 0 & 0 & 1 & 1 & 0 & $\begin{array}{l}(A 0, B 1),(B 0, A 1),(C 0, D 1),(D 0, C 1) \\
(A 1, B 0),(B 1, A 0),(C 1, D 0),(D 1, C 0)\end{array}$ \\
\hline$S_{7}$ & $\begin{array}{l}0 \\
\\
1\end{array}$ & 0 & 0 & 1 & 1 & 1 & $\begin{array}{l}(A 0, D 1),(B 0, C 1),(C 0, A 1),(D 0, B 1) \\
(A 1, D 0),(B 1, C 0),(C 1, A 0),(D 1, B 0)\end{array}$ \\
\hline$S_{8}$ & $\begin{array}{l}0 \\
1\end{array}$ & 0 & 1 & 0 & 0 & 0 & $\begin{array}{l}(A 0, A 2),(B 0, B 2),(C 0, C 2),(D 0, D 2) \\
(A 2, A 0),(B 2, B 0),(C 2, C 0),(D 2, D 0)\end{array}$ \\
\hline \begin{tabular}{|l|}
$S_{9}$ \\
\end{tabular} & $\begin{array}{l}0 \\
1\end{array}$ & 0 & 1 & 0 & 0 & 1 & $\begin{array}{l}(A 0, C 2),(B 0, D 2),(C 0, B 2),(D 0, A 2) \\
(A 2, C 0),(B 2, D 0),(C 2, B 0),(D 2, A 0)\end{array}$ \\
\hline$S_{10}$ & $\begin{array}{l}0 \\
1\end{array}$ & 0 & 1 & 0 & 1 & 0 & $\begin{array}{l}(A 0, B 2),(B 0, A 2),(C 0, D 2),(D 0, C 2) \\
(A 2, B 0),(B 2, A 0),(C 2, D 0),(D 2, C 0)\end{array}$ \\
\hline$S_{11}$ & $\begin{array}{l}0 \\
1\end{array}$ & 0 & 1 & 0 & 1 & 1 & $\begin{array}{l}(A 0, D 2),(B 0, C 2),(C 0, A 2),(D 0, B 2) \\
(A 2, D 0),(B 2, C 0),(C 2, A 0),(D 2, B 0)\end{array}$ \\
\hline$S_{12}$ & $\begin{array}{l}0 \\
1\end{array}$ & 0 & 1 & 1 & 0 & 0 & $\begin{array}{l}(A 0, A 3),(B 0, B 3),(C 0, C 3),(D 0, D 3) \\
(A 3, A 0),(B 3, B 0),(C 3, C 0),(D 3, D 0)\end{array}$ \\
\hline$S_{13}$ & $\begin{array}{l}0 \\
1\end{array}$ & 0 & 1 & 1 & 0 & 1 & $\begin{array}{l}(A 0, C 3),(B 0, D 3),(C 0, B 3),(D 0, A 3) \\
(A 3, C 0),(B 3, D 0),(C 3, B 0),(D 3, A 0) \\
\end{array}$ \\
\hline$S_{14}$ & $\begin{array}{l}0 \\
1\end{array}$ & 0 & 1 & 1 & 1 & 0 & $\begin{array}{l}(A 0, B 3),(B 0, A 3),(C 0, D 3),(D 0, C 3) \\
(A 3, B 0),(B 3, A 0),(C 3, D 0),(D 3, C 0)\end{array}$ \\
\hline$S_{15}$ & $\begin{array}{l}0 \\
1\end{array}$ & 0 & 1 & 1 & 1 & 1 & $\begin{array}{l}(A 0, D 3),(B 0, C 3),(C 0, A 3),(D 0, B 3) \\
(A 3, D 0),(B 3, C 0),(C 3, A 0),(D 3, B 0)\end{array}$ \\
\hline$S_{16}$ & $\begin{array}{l}0 \\
1\end{array}$ & 1 & 0 & 0 & 0 & 0 & $\begin{array}{l}(A 1, A 2),(B 1, B 2),(C 1, C 2), \\
(A 2, A 1),(B 2, B 1),(C 2, C 1),(D 2, D 1)\end{array}$ \\
\hline$S_{17}$ & $\begin{array}{l}0 \\
1\end{array}$ & 1 & 0 & 0 & 0 & 1 & 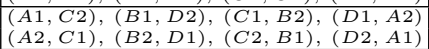 \\
\hline$S_{18}$ & $\begin{array}{l}0 \\
1\end{array}$ & 1 & 0 & 0 & 1 & 0 & 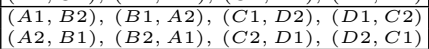 \\
\hline$S_{19}$ & $\begin{array}{l}0 \\
1\end{array}$ & 1 & 0 & 0 & 1 & 1 & 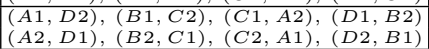 \\
\hline$S_{20}$ & $\begin{array}{l}0 \\
1\end{array}$ & 1 & 0 & 1 & 0 & 0 & $\begin{array}{l}(A 1, A 3),(B 1, B 3),(C 1, C 3), \\
(A 3, A 1),(B 3, B 1),(C 3, C 1),(D 3, D 1)\end{array}$ \\
\hline$S_{21}$ & $\begin{array}{l}0 \\
1\end{array}$ & 1 & 0 & 1 & 0 & 1 & $\begin{array}{l}(A 1, C 3)(B 1, D 3),(C 1, B 3),(D 1, A 3) \\
(A 3, C 1),(B 3, D 1),(C 3, B 1),(D 3, A 1)\end{array}$ \\
\hline$S_{22}$ & $\begin{array}{l}0 \\
1\end{array}$ & 1 & 0 & 1 & 1 & 0 & 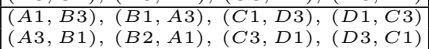 \\
\hline$S_{23}$ & $\begin{array}{l}0 \\
1\end{array}$ & 1 & 0 & 1 & 1 & 1 & $\begin{array}{l}(A 1, D 3),(B 1, C 3),(C 1, A 3),(D 1, B 3) \\
(A 3, D 1),(B 3, C 1),(C 3, A 1),(D 3, B 1)\end{array}$ \\
\hline$S_{24}$ & $\begin{array}{l}0 \\
1\end{array}$ & 1 & 1 & 0 & 0 & 0 & 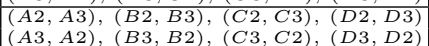 \\
\hline$S_{25}$ & $\begin{array}{l}0 \\
1\end{array}$ & 1 & 1 & 0 & 0 & 1 & 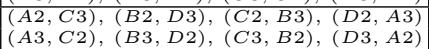 \\
\hline$S_{26}$ & $\begin{array}{l}0 \\
1\end{array}$ & 1 & 1 & 0 & 1 & 0 & $\begin{array}{l}(A 2, B 3),(B 2, A 3),(C 2, D 3),(D 2, C 3) \\
(A 3, B 2),(B 3, A 2),(C 33, D 2),(D 3, C 2)\end{array}$ \\
\hline$S_{27}$ & $\begin{array}{l}0 \\
1\end{array}$ & 1 & 1 & 0 & 1 & 1 & 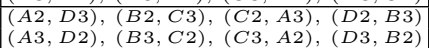 \\
\hline$S_{28}$ & $\begin{array}{l}0 \\
1\end{array}$ & 1 & 1 & 1 & 0 & 0 & $\begin{array}{l}(A 2, A 2),(B 2, B 2),(C 2, C 2),(D 2, D 2) \\
(A 3, A 3),(B 3, B 3),(C 3, C 3),(D 3, D 3)\end{array}$ \\
\hline$S_{29}$ & $\begin{array}{l}0 \\
1\end{array}$ & 1 & 1 & 1 & 0 & 1 & 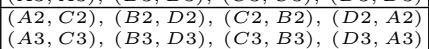 \\
\hline$S_{30}$ & $\begin{array}{l}0 \\
1\end{array}$ & 1 & 1 & 1 & 1 & 0 & $\begin{array}{l}(A 2, B 2),(B 2, A 2),(C 2, D 2),(D 2, C 2) \\
(A 3, B 3),(B 3, A 3),(C 3, D 3),(D 3, C 3)\end{array}$ \\
\hline$S_{31}$ & $\begin{array}{l}0 \\
1\end{array}$ & 1 & 1 & 1 & 1 & 1 & 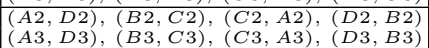 \\
\hline
\end{tabular}

where the left number of each block is the index $m 1$ of the subset $S_{m 1}^{(1)}$, which contains the 4-D point sent by Antenna 1, and the right number is the index $m 2$ of the subset $S_{m 2}^{(2)}$, which contains the 4-D point sent by Antenna 2 at discrete time $n$. To see that this block code is optimal in the sense of product distance, compare the Figs. 1 and 6 that are identical except for the point labeling. Looking now to Table 1, it is not difficult to check that, if the block code of length 2 defined over the alphabet 16-QAM is optimal in the sense of product distance, as is asserted in [1], then our 4-D code is as well.

Eight bits are needed to select one out of 256 points of the 4-D signal constellation. The blocks with eight bits are constructed as follows: The first five bits select one out of 32 subsets $S_{m}(m=0,1, \ldots, 31)$, and the other three bits choose one out of the eight 4-D points of the selected subset. This means that a 4-D point can carry eight bits of information with no error control. In order to improve the reliability of the wireless 


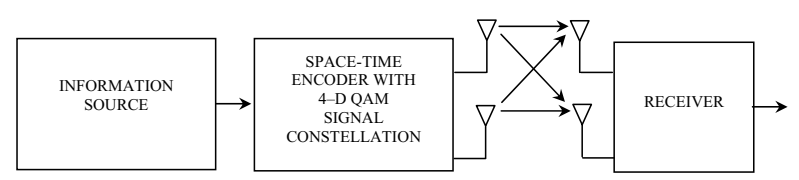

Fig. 7. Space-time coded modulation chain with two transmit antennas and two receive antennas.

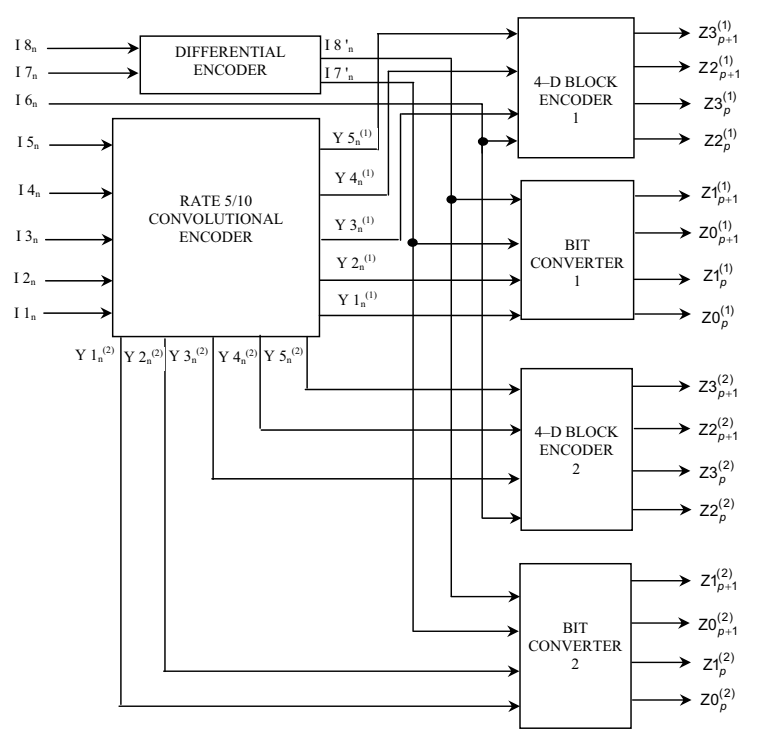

Fig. 8. General structure of a ST coded modulation transmitter with two antennas for sending 4 bits per signaling interval.

communication, we will use two transmit antennas instead of a single one. The receiver can use any number of antennas, but in our simulations we only considered the cases of a single antenna and of two antennas. This approach is called antenna diversity. Note that there is no need to enlarge the bandwidth of the radio channel. The transmission chain is shown in Fig. 7.

Suppose now that the information source delivers bits serially under a clock with a period of $T_{b}$ seconds. Then, the bit stream is divided into eight bit blocks, what is tantamount to a serial to parallel conversion under a clock with period $T=8 T_{b}$. Denote the eight bits of the $n$-th block as $I 1_{n}, \ldots, I 8_{n}$. As shown in Fig. 8, the first five bits $I 1_{n}, \ldots, I 5_{n}$ enter a rate $5 / 10$ convolutional encoder that outputs two groups of five bits $Y 1^{(i)}, Y 2^{(i)}, Y 3^{(i)}, Y 4^{(i)}$, and $Y 5^{(i)}(i=1,2)$. The first group (for $i=1$ ) selects a 4-D subset, a point of which will be transmitted from the Antenna 1, and the second group (for $i=2$ ) will feed similarly the Antenna 2. Every antenna transmits successively the two constituent 2-D points of a 4-D point using the classical QAM scheme.

With this notation, the decimal value of the index $m$ of the subset $S_{m}$ is given by

$$
m=16 Y 5^{(i)}+8 Y 4^{(i)}+4 Y 3^{(i)}+2 Y 2^{(i)}+Y 1^{(i)} .
$$

To determine a 2-D point from the signal constellation shown in Fig. 6, four bits are needed. Denote as $Z 0_{p}^{(i)}, Z 1_{p}^{(i)}, Z 2_{p}^{(i)}$, and $Z 3_{p}^{(i)}, i=1,2$, the four bits that select the first constituent 2-D point of the 4-D point transmitted by the antenna $i(i=$ $1,2)$ and as $Z 0_{p+1}^{(i)}, Z 1_{p+1}^{(i)}, Z 2_{p+1}^{(i)}$, and $Z 3_{p+1}^{(i)}, i=1,2$, the
Table 2. Correspondence between $Z 1_{l}^{(i)} Z 0_{l}^{(i)}$ and the 2-D four subsets.

\begin{tabular}{|c|c|c|}
\hline 2-D subset & $Z 1_{l}^{(i)}$ & $Z 0_{l}^{(i)}$ \\
\hline$A$ & 0 & 0 \\
\hline$B$ & 1 & 0 \\
\hline$C$ & 0 & 1 \\
\hline$D$ & 1 & 1 \\
\hline
\end{tabular}

Table 3. Correspondence between $Z 3_{k}^{(i)} Z 2_{k}^{(i)}$ and the four rings.

\begin{tabular}{|c|c|c|}
\hline Ring & $Z 3_{k}^{(i)}$ & $Z 2_{k}^{(i)}$ \\
\hline$R_{0}$ & 0 & 0 \\
\hline$R_{1}$ & 0 & 1 \\
\hline$R_{2}$ & 1 & 0 \\
\hline$R_{3}$ & 1 & 1 \\
\hline
\end{tabular}

four bits that select the second constituent 2-D point of the 4-D point transmitted by the same antenna $i$. The correspondence between $Z 1_{l}^{(i)} Z 0_{l}^{(i)}, l=p, p+1$, and the four 2-D subsets $A, B, C, D$ is as given in Table 2 . The correspondence between $Z 3_{k}^{(i)} Z 2_{k}^{(i)}, k=p$ and $p+1$, and the four rings of the 2-D signal constellation of Fig. 6 is as given in Table 3 .

Remember that, by the way the 4-D signal constellation has been partitioned, its 32 subsets $S_{i}, i=1, \ldots, 31$, are invariant to rotations by multiples of $90^{\circ}$. However, the eight points inside every subset are not. In order to make the scheme transparent to all the phase ambiguities of the signal constellation, we differentially encode the bit pair $I 8_{n} I 7_{n}$. Note that $I 7_{n}$ and $I 8_{n}$ are two of the three uncoded bits that select a 4-D point inside a given subset $S_{i}$. Therefore, we translate a sequence of this pair by the same number of positions (zero, one, two, or three) into a circular sequence, $00,01,10,11$. As a result, the sequence of 2-D points produced by the 4-D constellation mapping procedure will be rotated by $0^{\circ}, 90^{\circ}, 180^{\circ}$, and $270^{\circ}$ clockwise, respectively. Hence, a differential encoder (see Fig. 8) of the form

$$
I 8_{n}^{\prime} I 7_{n}^{\prime}=\left(I 8_{n-1}^{\prime} I 7_{n-1}^{\prime}+I 8_{n} I 7_{n}\right) \quad \bmod 100_{\text {base } 2} .
$$

and the corresponding differential decoder of the form

$$
I 8_{n} I 7_{n}=\left(I 8_{n}^{\prime} I 7_{n}^{\prime}-I 8_{n-1}^{\prime} I 7_{n-1}^{\prime}\right) \quad \bmod 100_{\text {base } 2} .
$$

at the output of the trellis decoder will remove all the phase ambiguities of the signal constellation.

The bit converter 1 and the bit converter 2 (see Fig. 8) are identical and each one converts the four bits $Y 1^{(i)}, Y 2^{(i)}, I 7_{n}^{\prime}$ and $I 8_{n}^{\prime}, i=1,2$, into two pairs of selection bits $Z 1_{p}^{(i)} Z 0_{p}^{(i)}$ and $Z 1_{p+1}^{(i)} Z 0_{p+1}^{(i)}$, which are used to select the pair of 2-D subsets corresponding to the 4-D type. With the correspondence between the bit pair $Z 1_{l}^{(i)} Z 0_{l}^{(i)}$ and the 2-D subsets $A, B, C, D$ as shown in Table 2, the operation of the bit converter is represented in Table 4.

The 4-D block encoder 1 and the 4-D block encoder 2 of Fig. 8 are also identical. Each of them takes four bits $Y 3^{(i)}$, 
Table 4. Partition of the 4-D 256 point rectangular constellation into 16 types.

\begin{tabular}{|cc|cc|c|cc|lc|}
\hline$I 8_{n}^{\prime} I 7_{n}^{\prime}$ & $Y 2^{(i)} Y 1^{(i)}$ & $\begin{array}{c}\text { 4-D } \\
\text { Types }\end{array}$ & $Z 1_{p}^{(i)} Z 0_{p}(i)$ & $Z 1_{p+1}^{(i)} Z 0_{p+1}^{(i)}$ \\
\hline 0 & 0 & 0 & 0 & A,A & 0 & 0 & 0 & 0 \\
0 & 1 & & & C,C & 0 & 1 & 0 & 1 \\
1 & 0 & & & B,B & 1 & 0 & 1 & 0 \\
1 & 1 & & & D,D & 1 & 1 & 1 & 1 \\
\hline 0 & 0 & 0 & 1 & A,C & 0 & 0 & 0 & 1 \\
0 & 1 & & & C,B & 0 & 1 & 1 & 0 \\
1 & 0 & & & B,D & 1 & 0 & 1 & 1 \\
1 & 1 & & & D,A & 1 & 1 & 0 & 0 \\
\hline 0 & 0 & 1 & 0 & A,B & 0 & 0 & 1 & 0 \\
0 & 1 & & & C,D & 0 & 1 & 1 & 1 \\
1 & 0 & & & B,A & 1 & 0 & 0 & 0 \\
1 & 1 & & & D,C & 1 & 1 & 0 & 1 \\
\hline 0 & 0 & 1 & 1 & A,D & 0 & 0 & 1 & 1 \\
0 & 1 & & & C,A & 0 & 1 & 0 & 0 \\
1 & 0 & & & B,C & 1 & 0 & 0 & 1 \\
1 & 1 & & & D,B & 1 & 1 & 1 & 0 \\
\hline
\end{tabular}

Table 5. The 4-D block encoders.

\begin{tabular}{|c|c|c|c|c|c|c|c|c|}
\hline$Y 5^{(i)}$ & $Y 4^{(i)}$ & $Y 3^{(i)}$ & $I 6_{n}$ & $\begin{array}{c}4-\mathrm{D} \\
\text { Shell }\end{array}$ & $Z 3_{p}^{(i)}$ & $Z 2_{p}^{(i)}$ & $Z 3_{p+1}^{(i)}$ & $Z 2_{p+1}^{(i)}$ \\
\hline 0 & 0 & 0 & 0 & $R_{0}, R_{0}$ & 0 & 0 & 0 & 0 \\
\hline 0 & 0 & 0 & 1 & $R_{1}, R_{1}$ & 0 & 1 & 0 & 1 \\
\hline 0 & 0 & 1 & 0 & $R_{0}, R_{1}$ & 0 & 0 & 0 & 1 \\
\hline 0 & 0 & 1 & 1 & $R_{1}, R_{0}$ & 0 & 1 & 0 & 0 \\
\hline 0 & 1 & 0 & 0 & $R_{0}, R_{2}$ & 0 & 0 & 1 & 0 \\
\hline 0 & 1 & 0 & 1 & $R_{2}, R_{0}$ & 1 & 0 & 0 & 0 \\
\hline 0 & 1 & 1 & 0 & $R_{0}, R_{3}$ & 0 & 0 & 1 & 1 \\
\hline 0 & 1 & 1 & 1 & $R_{3}, R_{0}$ & 1 & 1 & 0 & 0 \\
\hline 1 & 0 & 0 & 0 & $R_{1}, R_{2}$ & 0 & 1 & 1 & 0 \\
\hline 1 & 0 & 0 & 1 & $R_{2}, R_{1}$ & 1 & 0 & 0 & 1 \\
\hline 1 & 0 & 1 & 0 & $R_{1}, R_{3}$ & 0 & 1 & 1 & 1 \\
\hline 1 & 0 & 1 & 1 & $R_{3}, R_{1}$ & 1 & 1 & 0 & 1 \\
\hline 1 & 1 & 0 & 0 & $R_{2}, R_{3}$ & 1 & 0 & 1 & 1 \\
\hline 1 & 1 & 0 & 1 & $R_{3}, R_{2}$ & 1 & 1 & 1 & 0 \\
\hline 1 & 1 & 1 & 0 & $R_{2}, R_{2}$ & 1 & 0 & 1 & 0 \\
\hline 1 & 1 & 1 & 1 & $R_{3}, R_{3}$ & 1 & 1 & 1 & 1 \\
\hline
\end{tabular}

$Y 4^{(i)}, Y 5^{(i)}$, and $I 6_{n}, i=1,2$, and generates two pairs of selection bits $Z 2_{p}^{(i)} Z 3_{p}^{(i)}$ and $Z 2_{p+1}^{(i)} Z 3_{p+1}^{(i)}$, in accordance with Table 5.

As it may be seen from Table 1, every one of the 32 subsets contains eight 4-D points that are differen $\equiv$ any other one in both the first and the second 2-D componetrof it.

\section{4-D ROTATIONALLY INVARIANT ST TRELLIS CODES}

We will design now 32- and 64-states convolutional encoders, both linear and nonlinear, which fit in the general diagram of the ST coded modulation scheme shown in Fig. 8.

\section{A. 32-State Convolutional Encoders}

Denote the current state of the convolutional encoder as $W 5_{n} W 4_{n} W 3_{n} W 2_{n} W 1_{n}$, where $W 1_{n}, W 2_{n}, W 3_{n}, W 4_{n}$, and $W 5_{n}$ are binary variables. Let us number in decimal notation the states from 0 to 31 using the relation

$$
W_{n}=16 W 5_{n}+8 W 4_{n}+4 W 3_{n}+2 W 2_{n}+W 1_{n} .
$$

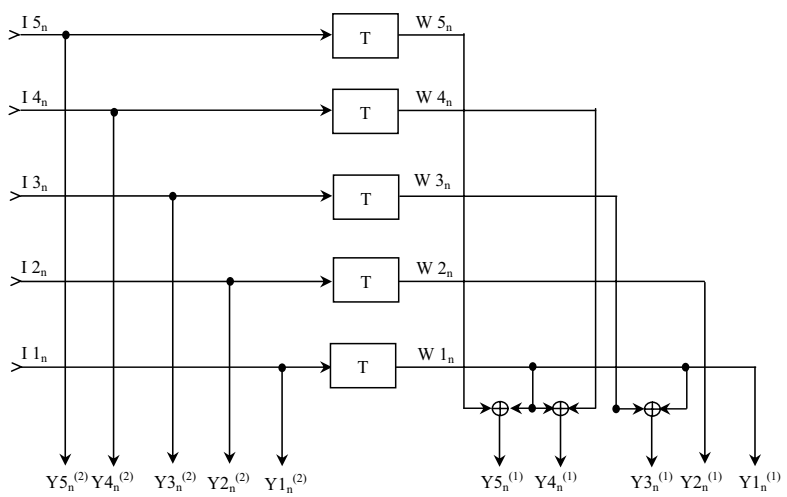

Fig. 9. 32-state, rate 5/10, 4-D linear convolutional encoder.

Using the rules 1 and 2 to label the state transitions, we obtain a linear code as follows. The first part of the label of all 32 transitions originating from the same state $W_{n}$ [see (23)] is as given by

$$
\begin{aligned}
Y 1_{n}^{(1)} & =W 1_{n} \\
Y 2_{n}^{(1)} & =W 2_{n} \\
Y 3_{n}^{(1)} & =W 1_{n} \oplus W 3_{n} \\
Y 4_{n}^{(1)} & =W 1_{n} \oplus W 4_{n} \\
Y 5_{n}^{(1)} & =W 1_{n} \oplus W 5_{n} .
\end{aligned}
$$

The second part of the label of the transition from the current state $W_{n}$ to the next state $W_{n+1}$ produced by the current 5-tuple of bits applied at the input is

$$
\begin{aligned}
Y 1_{n}^{(2)} & =I 1_{n} \\
Y 2_{n}^{(2)} & =I 2_{n} \\
Y 3_{n}^{(2)} & =I 3_{n} \\
Y 4_{n}^{(2)} & =I 4_{n} \\
Y 5_{n}^{(2)} & =I 5_{n} .
\end{aligned}
$$

The block diagram of this 32 states linear convolutional encoder is given in Fig. 9.

Consider next a different labeling of the state transitions such that the transitions arriving at the same state differ in both the first and the second symbol. The first part of the label of all 32 transitions originating from the same state $W_{n}$ is identical to that of the linear code. For the second part of the label, define first the decimal number $I_{n}$ as

$$
I_{n}=16 I 5_{n}+8 I 4_{n}+4 I 3_{n}+2 I 2_{n}+I 1_{n} .
$$

The second part of the label of the transition from the current state $W_{n}$ to the next state $W_{n+1}$ produced by the current 5-tuple of bits applied at the input is

$$
Y_{n}^{(2)}=W_{n}+I_{n} \quad(\text { modulo } 32) .
$$

The block diagram of the corresponding (nonlinear) trellis encoder is presented in Fig. 10.

In all designed 4-D ST codes, we used the rule 2 in that sense that the transitions merging in a state differ in both the first and the second symbol. 


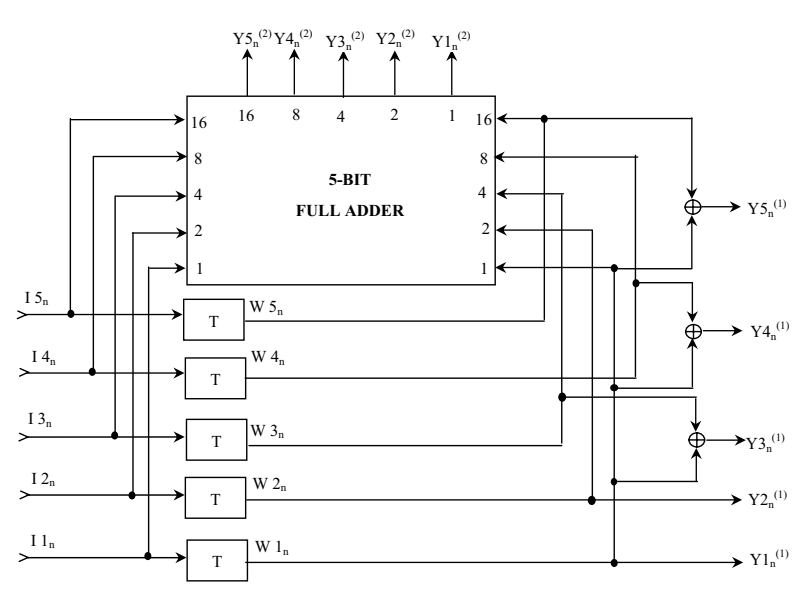

Fig. 10. 32-state, rate 5/10, 4-D ST nonlinear convolutional encoder.

\section{B. 64-State Nonlinear Encoder}

Denote the current state of the convolutional encoder as $W 6_{n} W 5_{n} W 4_{n} W 3_{n} W 2_{n} W 1_{n}$, where $W 1_{n}, W 2_{n}, W 3_{n}$, $W 4_{n}, W 5_{n}$, and $W 6_{n}$ are binary variables. Let us number in decimal notation the states from 0 to 63 using the relation

$$
\begin{aligned}
W_{n}= & 32 W 6_{n}+16 W 5_{n} \\
& +8 W 4_{n}+4 W 3_{n}+2 W 2_{n}+W 1_{n} .
\end{aligned}
$$

The first part of the label of all 32 transitions originating from the same state $W_{n}$ [see (28)] is as given below:

$$
\begin{aligned}
Y 1_{n}^{(1)} & =W 1_{n} \oplus W 6_{n} \\
Y 2_{n}^{(1)} & =W 2_{n} \\
Y 3_{n}^{(1)} & =W 1_{n} \oplus W 3_{n} \oplus W 6_{n} \\
Y 4_{n}^{(1)} & =W 1_{n} \oplus W 4_{n} \oplus W 6_{n} \\
Y 5_{n}^{(1)} & =W 1_{n} \oplus W 5_{n} \oplus W 6_{n} .
\end{aligned}
$$

For the second part of the label of a state transition, first form the following binary variables:

$$
\begin{aligned}
V 1_{n} & =W 1_{n} \oplus W 6_{n} \\
V 2_{n} & =W 2_{n} \\
V 3_{n} & =W 3_{n} \\
V 4_{n} & =W 4_{n} \\
V 5_{n} & =W 5_{n} .
\end{aligned}
$$

Now, with the binary variables $V 1_{n}, V 2_{n}, V 3_{n}, V 4_{n}$, and $V 5_{n}$ form the decimal number $V_{n}$ :

$$
V_{n}=16 V 5_{n}+8 V 4_{n}+4 V 3_{n}+2 V 2_{n}+V 1_{n} .
$$

The second part of the label of the transition from the current state $W_{n}$ to the next state $W_{n+1}$ produced by the current 5-tuple of bits applied at the input is then as written below:

$$
Y_{n}^{(2)}=V_{n}+I_{n} \quad(\text { modulo } 32) .
$$

The block diagram of this 64-state convolutional encoder is shown in Fig. 11.

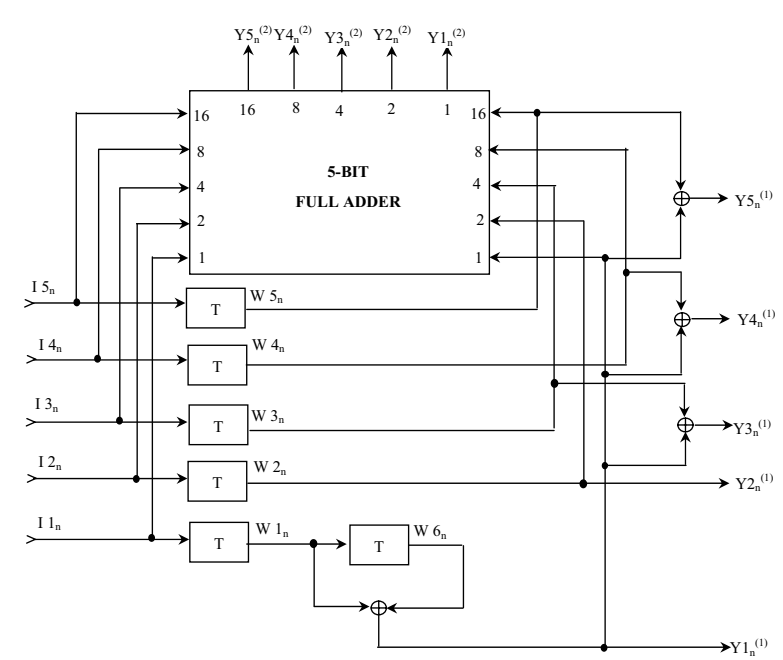

Fig. 11. 64-state, rate 5/10, 4-D ST nonlinear convolutional encoder.

\section{ERROR PERFORMANCE SIMULATIONS}

In this section, we first describe the transmission model as well as a typical MIMO channel model by using the complex baseband notation. Here, the ST coded modulation system shown in Fig. 7 is considered for wireless downlink transmission. It consists of two transmit antennas at the base station and one or two receive antennas at the mobile station.

We write the discrete time $n T$ simply as $n$, where $T$ is the 4-D symbol interval. Then, the 2-D symbol has $T / 2$ seconds duration and we write $2 p$ for $2 p \cdot T / 2$ and $2 p+1$ for $(2 p+1) \cdot T / 2$.

At the beginning and the end of each frame transmission, the convolutional encoder is required to be in state zero. Suppose now that at the discrete time $n$, the encoder is in state $W_{n}$ and is fed with a new block of eight information bits $I 1_{n}, \ldots, I 8_{n}$. According to the trellis diagram, this will make the encoder move into a new state $W_{n+1}$. The state transition $W_{n} \rightarrow W_{n+1}$ shows by its label $S_{n}^{(1)} S_{n}^{(2)}$ which 4-D subset is selected for transmission. In fact, however, not subsets but symbols within these subsets are transmitted. We then use $P_{n}^{(1)}$ and $P_{n}^{(2)}$ to denote 4-D symbols selected within their corresponding subsets $S_{n}^{(1)}$ and $S_{n}^{(2)}$, respectively, by the same group of three uncoded information bits $I 6_{n}, I 7_{n}^{\prime}$, and $I 8_{n}^{\prime}$. Now, both these symbols will be simultaneously transmitted in two successive 2-D time intervals of length $T / 2$.

Let us write $P_{n}^{(1)}=\left(p_{2 p}^{(1)}, p_{2 p+1}^{(1)}\right)$ and $P_{n}^{(2)}=\left(p_{2 p}^{(2)}, p_{2 p+1}^{(2)}\right)$. At time $2 p$, Antenna 1 and Antenna 2 will transmit the first 2-D points $p_{2 p}^{(1)}$ and $p_{2 p}^{(2)}$, respectively. After $T / 2$ seconds, at time $2 p+1$, Antenna 1 will transmit the second 2-D point $p_{2 p+1}^{(1)}$ and Antenna 2 will transmit the second 2-D point $p_{2 p+1}^{(2)}$.

At the receiver, the signal $r_{l}^{(j)},(l=2 p, 2 p+1)$ received by antenna $j(j=1,2)$ at time $l$ is given by

$$
r_{l}^{(j)}=\sum_{i=l}^{2} \alpha_{i j} \cdot p_{l}^{(i)} \cdot \sqrt{E_{s}}+\eta_{l}^{(j)} .
$$

where the noise $\eta_{l}^{(j)}$ at time $l=2 p, 2 p+1$ is modeled as independent samples of a zero-mean complex Gaussian random 
variable with variance $N_{0} / 2$ per dimension. In (33), $\sqrt{E_{s}}$ is a scale factor chosen so that the average energy of the signal constellation equals 1 and is the same as for the 2-D case as given by (18). Thus, $E_{s}=10$. In (33), the coefficient $\alpha_{i j}$ is the path gain from transmit antenna $i(i=1,2)$ to receive antenna $j(j=1,2)$. Under the assumption that the signals transmitted from different antennas undergo independent Rayleigh fading, the path gains $\alpha_{i j}$ can be modeled as independent samples of complex Gaussian random variables having mean zero and variance 0.5 per dimension. It is also assumed that the fading is quasi-static, which implies that the path gains are constant during a frame and vary independently from one frame to another. Several modeling methods have extensively been investigated in [25] to enable the efficient generation of multiple sequences of Rayleigh fading processes uncorrelated in time and between sequences. In this paper, we have used the so-called RANDN method [25].

To decode the proposed ST trellis code, we have used the Viterbi algorithm. Assuming ideal channel state information (CSI), the decoder has full knowledge of the path gains $\alpha_{i j}$ for $i=1,2$, and $j=1,2$. With two receive antennas, the branch metric for a state transition labeled by $S_{n}^{(1)} S_{n}^{(2)}$ is given by

$$
\sum_{l=1}^{2} \sum_{j=1}^{2}\left|r_{l}^{(j)}-\sum_{i=1}^{2} \alpha_{i j} \cdot p_{l}^{(i)}\right|^{2} .
$$

If only one receive antenna is used, (34) will be reduced to

$$
\sum_{l=1}^{2}\left|r_{l}-\sum_{i=1}^{2} \alpha_{i} \cdot p_{l}^{(i)}\right|^{2}
$$

According to the Viterbi algorithm, the path with the smallest accumulated metric will be selected as the decoding output.

Next, we provide simulation results for the performance of the ST codes, both 2-D and 4-D, given in Section II and in Section $\mathrm{V}$, respectively. Figs. 12 and 13 show frame error rates (FERs) of the developed 4-D QAM ST codes at rate $4 \mathrm{~b} / \mathrm{s} / \mathrm{Hz}$ with two transmit antennas and with one and two receive antennas, respectively. Figs. 14 and 15 show bit error rates (BERs). In these simulations, each frame consists of 654 -D symbols out of each transmit antenna. Note that the duration of 654 -D symbols equals the time length of $1302-\mathrm{D}$ symbols.

As seen from Figs. 12 and 13, the best code appears to be the 2-D nonlinear 64-state ST code, and the worst one, the 2-D nonlinear 16-state ST code, with all others in between, for both one and two receive antennas. This result would suggest that linear ST codes are preferable to their nonlinear counterparts. However, in case of 4-D codes, we can compare the 32-state linear and nonlinear and see that the latter one is always slightly better, for both FER and BER, and one or two antennas.

\section{CONCLUSIONS}

In our paper, we first presented a 16-state ST code that is different of that published in [1] and new 32- and 64-state ST codes using the same 16 QAM signal constellation. Then we considered a 4-D rectangular signal constellation whose 2-D constituent constellations are 16 QAM and we partitioned it into

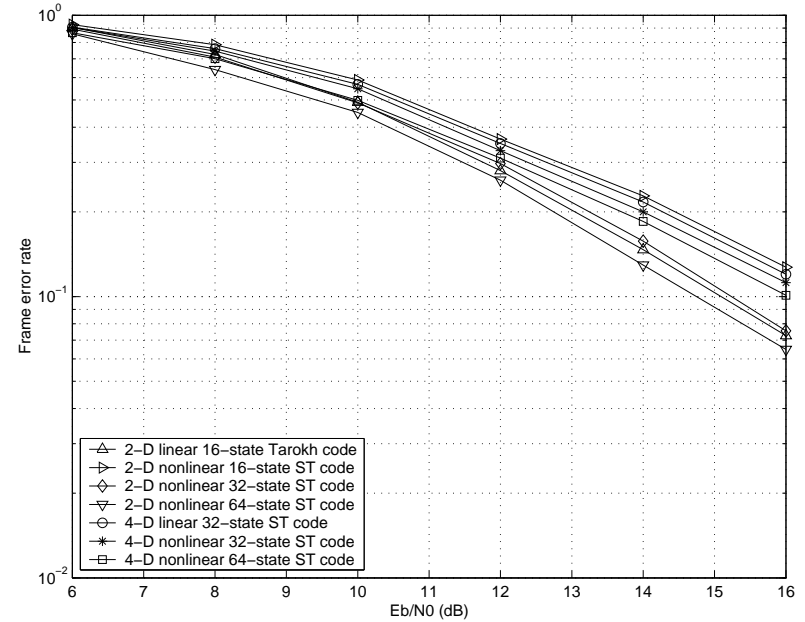

Fig. 12. The FER performance comparison of 2-D and 4-D QAM ST codes at $4 \mathrm{~b} / \mathrm{s} / \mathrm{Hz}$ with one receive antenna.

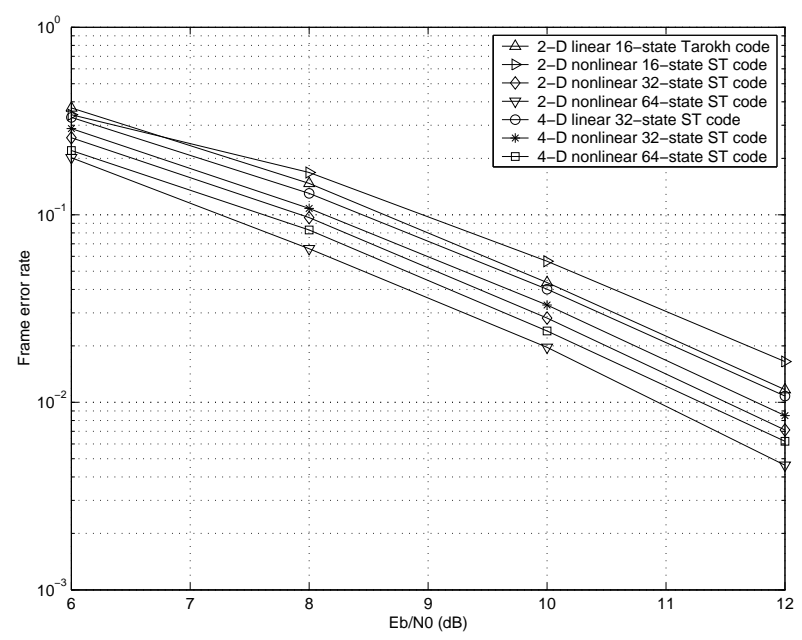

Fig. 13. The FER performance comparison of 2-D and 4-D QAM ST codes at $4 \mathrm{~b} / \mathrm{s} / \mathrm{Hz}$ with two receive antennas.

4-D subsets such that the points within a subset are different in both the first and the second 2-D component of it. We then constructed 4-D ST trellis codes that are fully rotationally invariant. For both the 2-D and 4-D ST codes, enlarging the number of states improves the FER performance, as expected. There is no important difference between the performance of 2-D and 4-D codes. The rotational invariance of the 4-D ST codes may contribute to alleviate the task of the carrier phase tracking circuit in the receiver.

Linear ST trellis codes are of course easier to design and to generate than their nonlinear counterparts. However, in case of 4-D at least, the results have confirmed our intuition that nonlinear ST codes could be better than the linear ones. Somebody skilled in the art may easily simplify our nonlinear ST codes, both 2-D and 4-D, to linear ones. The converse is clearly not true, and this is another reason why we have demonstrated mostly nonlinear ST codes in this paper.

Nonlinear codes perform slightly better than the linear ones of same complexity. This is explained by a better labelling of 


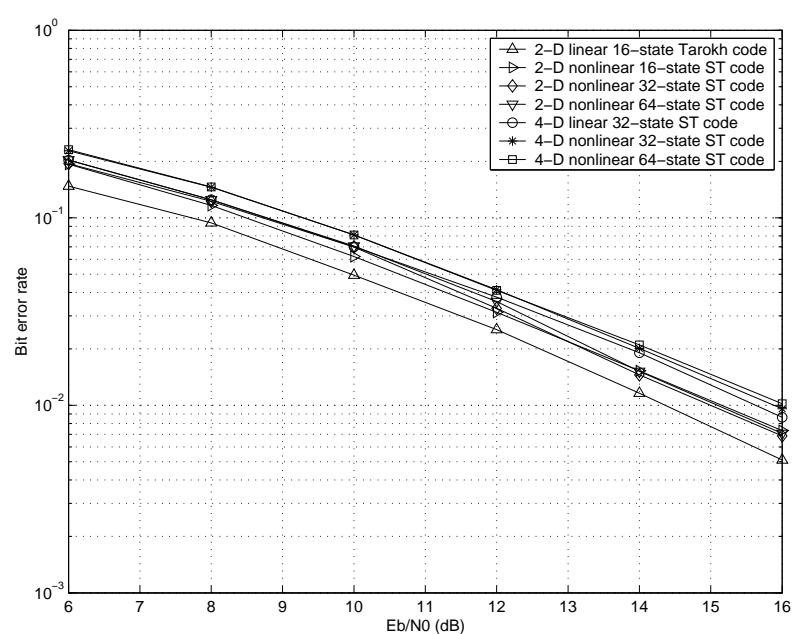

Fig. 14. The BER performance comparison of 2-D and 4-D QAM ST codes at $4 \mathrm{~b} / \mathrm{s} / \mathrm{Hz}$ with one receive antenna.

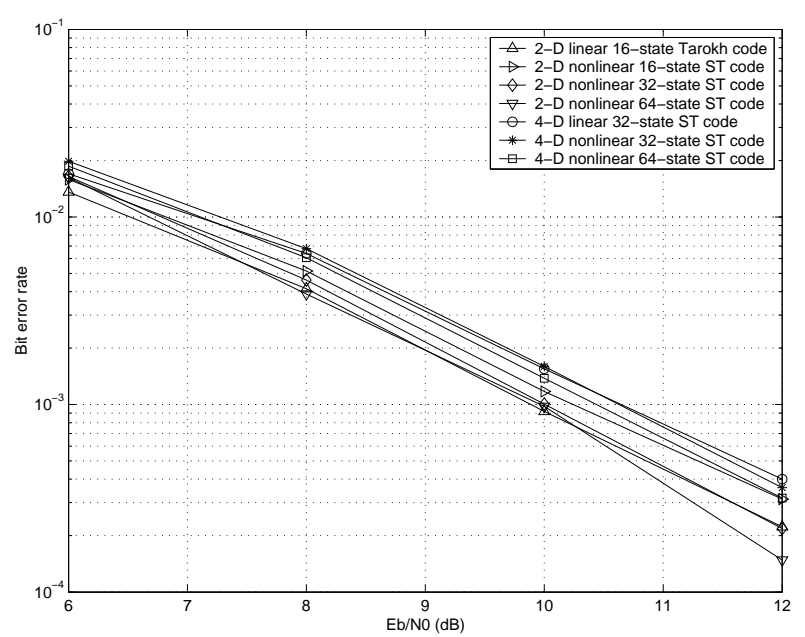

Fig. 15. The BER performance comparison of 2-D and 4-D QAM ST codes at $4 \mathrm{~b} / \mathrm{s} / \mathrm{Hz}$ with two receive antennas.

state transitions in the trellis diagram. Indeed, for linear codes, transitions originating in the same state and transitions joining in the same state differ only in one of the two symbols, simultaneously transmitted by the two antennas, which label the transitions, while the condition we impose on labels to differ in both the first and the second symbol results in nonlinear codes. The Hamming distance between labels of nonlinear codes is thus maximized, a desirable property as shown in [19].

\section{REFERENCES}

[1] V. Tarokh, N. Seshadri, and A. R. Calderbank, "Space-time codes for high data rate wireless communications: Performance criterion and code construction," IEEE Trans. Inform. Theory, vol. 44, pp. 744-765, Mar. 1998.

[2] A. F. Naguib, V. Tarokh, N. Seshadri, and A. R. Calderbank, "A spacetime coding modem for high data rate wireless communications," IEEE J. Select. Areas Commun., vol. 16, pp. 1459-1478, Oct. 1998.

[3] V. Tarokh, A. F. Naguib, N. Seshadri, and A. R. Calderbank, "Space-time codes for high data rate wireless communications: Performance criteria in the presence of channel estimation errors, mobility, and multiple paths," IEEE Trans. Commun., vol. 47, pp. 199-207, Feb. 1999.

[4] V. Tarokh, A. F. Naguib, N. Seshadri, and A. R. Calderbank, "Combined array processing and space-time coding," IEEE Trans. Inform. Theory, vol. 45, pp. 1121-1128, May 1999.
[5] A. F. Naguib, N. Seshadri, and A. R. Calderbank, "A space-time coding and signal processing for high data rate wireless communications," IEEE Signal Processing Magazine, vol. 17, pp. 76-92, May 2000.

[6] S. M. Alamouti, "A simple transmit diversity technique for wireless communications," IEEE J. Select. Areas Commun., vol. 16, pp. 1451-1458, Oct. 1998.

[7] H. Jafarkhani and N. Seshadri, "Super-orthogonal space-time trellis codes," IEEE Trans. Inform. Theory, vol. 49, pp. 937-950, Apr. 2003.

[8] B. M. Hochwald and T. L. Marzetta, "Unitary space-time modulation for multiple-antenna communications in Rayleigh flat fading," IEEE Trans. Inform. Theory, vol. 46, pp. 543-564, Mar. 2000.

[9] B. M. Hochwald and W. Sweldens, "Differential unitary space-time modulation," IEEE Trans. Commun., vol. 48, pp. 2041-2052, Dec. 2000.

[10] B. L. Hughes, "Differential space-time modulation," IEEE Trans. Inform. Theory, vol. 46, pp. 2567-2578, Nov. 2000.

[11] B. L. Hughes, "Optimal space-time constellations from groups," IEEE Trans. Inform. Theory, vol. 49, pp. 401-410, Feb. 2003.

[12] B. Hassibi and B. M. Hochwald, "How much training is needed in multiple-antenna wireless links?" IEEE Trans. Inform. Theory, vol. 49, pp. 951-963, Apr. 2003.

[13] E. Biglieri, J. Proakis, and S. Shamai, "Fading channels: Informationtheoretic and communications aspects," IEEE Trans. Inform. Theory, vol. 44, pp. 2619-2692, Oct. 1998.

[14] E. Biglieri, G. Caire, and G. Taricco, "Limiting performance of blockfading channels with multiple antennas," IEEE Trans. Inform. Theory, vol. 47, pp. 1273-1289, May 2001.

[15] M. Pätzold, Mobile Fading Channels. Chichester: John Wiley \& Sons, 2002.

[16] A. Burr, Modulation and Coding for Wireless Communications. New York: Prentice-Hall, 2001.

[17] B. Vucetic and J. Yuan, Space-Time Coding. Chichester: John Wiley \& Sons, 2003.

[18] H. El Gamal and M.O. Damen, "Universal space-time coding," IEEE Trans. Inform. Theory, vol. 49, pp. 1097-1119, May 2003.

[19] D. Divsalar and M. K. Simon, "The design of trellis coded MPSK for fading channels: Set partitioning for optimum code design," IEEE Trans. Commun., vol. 36, pp. 1013-1021, Sept. 1988.

[20] D. Divsalar and M. K. Simon, "Multiple trellis coded modulation (MTCM)," IEEE Trans. Commun., vol. 36, pp. 410-419, Apr. 1988.

[21] E. Biglieri, D. Divsalar, P. J. McLane, and M. K. Simon, Introduction to trellis-coded modulation with applications. New York: Macmillan Publishing Company, 1991.

[22] L. F. Wei, "Trellis-coded modulation with multidimensional constellations," IEEE Trans. Inform. Theory, vol. 33, pp. 483-501, July 1987.

[23] C. E. D. Sterian, F. Laue, and M. Pätzold, "Trellis-coded quadrature amplitude modulation with $2 \mathrm{~N}$-dimensional constellations for mobile radio channels," IEEE Trans. Veh. Technol., vol. 48, pp. 1475-1487, Sept. 1999.

[24] C. E. D. Sterian, "Wei-type trellis-coded modulation with $2 \mathrm{~N}$-dimensional rectangular constellation for $N$ not a power of two," IEEE Trans. Inform. Theory, vol. 43, pp. 750-758, Mar. 1997.

[25] C.-X. Wang and M. Pätzold, "Methods of generating multiple uncorrelated Rayleigh fading processes," in Proc. IEEE VTC 2003-Spring, Jeju, Korea, Apr. 2003, pp. 510-514.

[26] C. E. D. Sterian, "Exact formulas for computing the energy of "square" and "cross" two-dimensional rectangular signal constellations," European Trans. Telecommun., vol. 8, pp. 547-549, Nov./Dec. 1997.

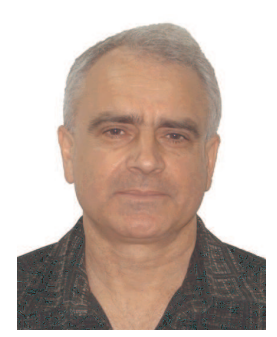

Corneliu Eugen D. Sterian was born in Bucharest, Romania, on April 30, 1947. He received the Dipl.Ing. and Dr.-Ing. degrees in electronics and telecommunications engineering from the University POLITEHNICA of Bucharest. He is currently Associate Professor with the University POLITEHNICA of Bucharest where he is teaching Information and Coding Theory, Communication Systems, and related matters. During the second half of the year 2000 he was the telecommunications engineer of UNMIK, Pristina, Kosovo. From the middle of 1997 to the end of 1998, he was Director General in the Ministry of Communications of his country. After the Ministry was replaced by the National Agency for Communications and Information Technology, he served as Head of Department and then Director. From 1992 to 1997, he was with ROMTELECOM, the national telecommunications operator of Romania. From 1974 to 1992, he was with I.P.A. research institute, where he was promoted in 1991 to the highest degree 
of principal scientist. His research interests include information theory and more particularly channel coding.

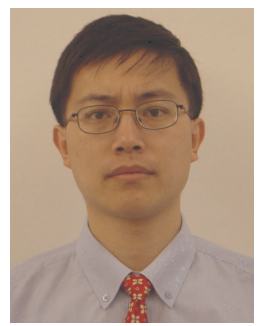

Cheng-Xiang Wang was born in Shandong, P. R. China, in 1975. He received the Bachelor of Science and Master of Engineering degrees in communication and information systems from Shandong University, Shandong, P. R. China, in 1997 and 2000, respectively. In 2001, he worked as a Student Research Assistant (Studentische Hilfskraft) in the Department of Communication Networks at the Technical University of Hamburg-Harburg, Hamburg, Germany. Since October 2001, he has been with Agder University College, Grimstad, Norway, and is currently working towards his Ph.D. degree at Aalborg University, Aalborg, Denmark. From January to April 2004, Mr. Wang was on leave at Baseband Algorithms \& Standardization Lab of Siemens AG-Mobile Phones, Munich, Germany, conducting research and development of error models for EGPRS systems within the framework of the 3GPP GERAN System Concept R\&D Project. Mr. Wang has published more than 30 papers in journals and conference proceedings. His current research interests include mobile fading channel modeling, error-correction techniques for mobile communications, especially error models, space-time coding, multilevel coded modulation techniques, and their applications in mobile communications. His master thesis was awarded "Excellent Master Thesis of Shandong Province in 2001". Together with Prof. Dong-Feng Yuan, Ms. Qi Yao, and other colleagues, he was awarded the third prize of "Scientific Improvements Prize of Shandong Province in 2000", in the virtue of the project "Research on Optimum Coded Modulation Schemes in Digital Mobile Data and Image Transmission Systems".

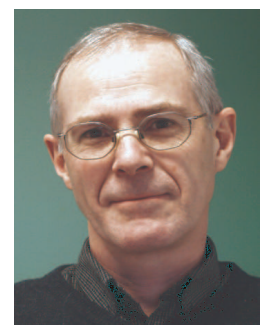

Ragnar Johnsen was born in Arendal, Norway, in 1949. He received the Master of Science in Electronics and Telecommunications from NTH in 1973. From 1974 to 1979, he worked for the Norwegian Telecom Research and Development Department and from 1979 to 1986 for the Norwegian Telecom Education and Training Center as a lecturer. Since 1986 he is employed with Agder University College in Grimstad, Norway, as a lecturer, giving courses in Telecommunications as well as Mobile Communications, Digital Signal Processing and Digital Electronics. Together with O.T. Aas he wrote the book"Fiber Optic Transmission", Universitetsforlaget AS Oslo, Oslo 1987. For Aschehoug og Gyldendals Store Norske Leksikon (encyclopedia) Kunnskapsforlaget 1995 to 1999, he was responsible for all material about Telecommunications, Electronic Consumer Products and Signal Processing.

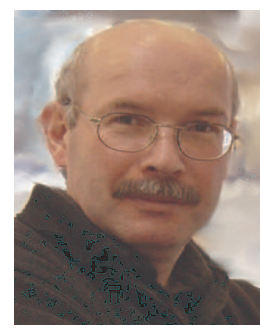

Matthias Pätzold was born in Engelsbach, Germany, in 1958. He received the Dipl.-Ing. and Dr.-Ing. degrees in electrical engineering from Ruhr-University Bochum, Bochum, Germany, in 1985 and 1989, respectively, and the habil. degree in communications engineering from the Technical University of Hamburg-Harburg, Hamburg, Germany, in 1998.

From 1990 to 1992, he was with ANT Nachrichtentechnik GmbH, Backnang, Germany, where he was engaged in digital satellite communications. From 1992 to 2001, he was with the department of digital networks at the Technical University Hamburg-Harburg. Since 2001, he has been a full professor of mobile communications with Agder University College, Norway. He is author of the books "Mobile Radio Channels - Modelling, Analysis, and Simulation" (in German) (Wiesbaden, Germany: Vieweg, 1999) and "Mobile Fading Channels" (Chichester, U.K.: Wiley, 2002). His current research interests include mobile radio communications, especially multipath fading channel modelling, multi-input multi-output (MIMO) systems, channel parameter estimation, and coded-modulation techniques for fading channels. Prof. Pätzold received the "1998 Neal Shepherd Memorial Best Propagation Paper Award" from the IEEE Vehicular Technology Society and was also the recipient of the "2002 Neal Shepherd Memorial Best Propagation Paper Award". He is the recipient of the "2003 Excellent Paper Award" of the IEEE Int. Symp. on Personal, Indoor and Mobile Radio Communications (PIMRC'03), Beijing, China 